\title{
Discrete Green's Functions
}

\author{
By G. T. McAllister and E. F. Sabotka
}

\begin{abstract}
Let $G(P ; Q)$ be the discrete Green's function over a discrete $h$-convex region $\Omega$ of the plane; i.e., $a(P) G_{x \bar{x}}(P ; Q)+c(P) G_{y \bar{y}}(P ; Q)=-\delta(P ; Q) / h^{2}$ for $P \in \Omega_{h}, G(P ; Q)=0$ for $P \in \partial \Omega_{h}$. Assume that $a(P)$ and $c(P)$ are Hölder continuous over $\Omega$ and positive. We show that $\left|D^{(m)} G(P ; Q)\right| \leqq A_{m} / \rho_{P Q}^{m}$ and $\left|\tilde{D}^{(m)} G(P ; Q)\right| \leqq B_{m} d(Q) / \rho_{P Q}^{m+1}$, where $D^{(m)}$ is an $m$ th order difference quotient with respect to the components of $P$ or $Q$, and $\tilde{D}^{(m)}$ denotes an $m$ th order difference quotient only with respect to the components of $P$.
\end{abstract}

Introduction. Place a square grid on the plane with grid width $h$; grid points are $P=(m h, n h)$ where $m$ and $n$ are integers. If $P=(x, y)$ is a grid point, the neighbors of $P$ are the points $P_{1}=(x+h, y), P_{2}=(x, y+h), P_{3}=(x-h, y)$, and $P_{4}=$ $(x, y-h)$.

Let $\Omega$ be a plane region. Let $\Omega_{h}$ be the set of grid points $P \in \Omega$ such that the four neighbors of $P$ are in $\Omega$. Let $\partial \Omega_{h}$ be that set of grid points in $\bar{\Omega}$ with at least one neighbor in the exterior of $\Omega$.

Let $W(P)$ be defined on $\Omega_{h}+\partial \Omega_{h}$. Let $P \in \Omega_{h}$. Then we make the following definitions: $h W_{x}(P)=W\left(P_{1}\right)-W(P), h W_{\hat{x}}(P)=W(P)-W\left(P_{3}\right), h W_{\nu}(P)=W\left(P_{2}\right)-$ $W(P), h W_{\bar{\nu}}(P)=W(P)-W\left(P_{4}\right), W_{x \dot{x}}(P)=\left(W_{x}(P)\right)_{\dot{x}}, W_{y \bar{y}}(P)=\left(W_{\nu}(P)\right)_{\hat{\nu}}$, and $W_{x y}(P)=\left(W_{x}(P)\right)_{y}$.

Let $a(P)$ and $c(P)$ be Hölder continuous functions on $\bar{\Omega}$; let $\lambda$ and $L$ denote the positive minimum and maximum of these functions over $\bar{\Omega}$. Let $Q \in \Omega_{h}$ and let $G(P ; Q)$ be the solution to the problem

$$
\begin{aligned}
a(P) G_{x \grave{x}}(P ; Q)+c(P) G_{y \dot{y}}(P ; Q) & =-\delta(P ; Q) / h^{2}, & & P \in \Omega_{h}, \\
G(P ; Q) & =0, & & P \in \partial \Omega_{h} ;
\end{aligned}
$$

here difference quotients are with respect to the components of $P$ and $\delta(P ; Q)$ is the Kronecker symbol.

In this paper, we obtain estimates on $D^{(m)} G(P ; Q)$ and $\tilde{D}^{(m)} G(P ; Q)$ where $D^{(m)}$ denotes an $m$ th order difference quotient with respect to the components of $P$ and/or $Q$ and $\tilde{D}^{(m)} G(P ; Q)$ denotes an $m$ th order difference quotient with respect to the components either of $P$ or of $Q$. Basic to our methods of obtaining these estimates is the discrete analogue of the logarithm function as developed by McCrea and Whipple [7].

A significance of estimates of the type carried out in this paper is that they may be used, as in [5], to obtain pointwise a priori estimates on difference quotients of solutions to linear difference equations and these estimates may be used for the construction, as in [6], of a solution to nonlinear difference equations. Such a priori

Received March 6, 1972.

AMS (MOS) subject classifications (1970). Primary 65N20.

Key words and phrases. Elliptic difference equations, finite differences. 
estimates on difference quotients are also important in showing the convergence of specific numerical methods, as in [2], and in showing that solutions of difference equations converge - often these estimates give an order of convergence-to a solution of the differential equation, as in [9] and [11].

Our results may also be used to obtain estimates on the difference between difference quotients of the discrete and of the continuous Green's function; e.g. [8] and [10].

In the first five sections, we consider $G(P ; Q)$ only for the discrete Laplacian, i.e., $a(P) \equiv c(P) \equiv 1$. In Section 1 , we obtain an estimate on $G(P ; Q)$ when $\Omega_{h}$ is a half-plane. Our estimates are of the type $\left|D^{(m)} G(P ; Q)\right| \leqq A_{m} / \rho_{P Q}^{m}$ and $\left|\tilde{D}^{(m)} G(P ; Q)\right|$ $\leqq B_{m} d(Q) / \rho_{P Q}^{m+1}$ (or $\left|\tilde{D}^{(m)} G(P ; Q)\right| \leqq C_{m} d(P) / \rho_{P Q}^{m+1}$ ) where $\rho_{P Q}^{2}$ is the squared distance from $P$ to $Q$ plus $h^{2}, d(X)$ is the distance from $X$ to the $\partial \Omega_{h}$, and $A_{m}, B_{m}$ and $C_{m}$ are absolute constants - explicitly computed-which are independent of $h$. Some of these estimates are similar to those in Widman [12] who considers the Green's function for the continuous problem. In the discrete case, there are intrinsic difficulties which are not present in the continuous theory; e.g. we may not use any mapping techniques for the discrete problem. In Section 2 and in Section 3, we construct $G(P ; Q)$ for an infinite strip and for a rectangular region. From this construction, we obtain the same type of estimates as in Section 1. As a consequence of these sections, we may construct the $G(P ; Q)$ associated with the discrete Laplacian whenever $\Omega$ is a half-plane, quarter-plane, eighth-plane, strip, triangle or rectangle.

We extend our estimates in Section 4 to general domains which are discrete $h$-convex (see the text for the definition). Here we discover that second-order difference quotients of $G(P ; Q)$ exhibit a singularity in the neighborhood of an obtuse corner. The order of the singularity is slightly worse than that predicted in [4] for the continuous theory.

In Section 5, we consider the general equation in $\left(^{*}\right)$ under the assumption that the coefficients $a(P)$ and $c(P)$ are $\alpha$-Hölder continuous over $\bar{\Omega}$. These results represent an extension and an improvement of those in [5].

Some of our estimates implicitly require that the mesh size $h$ be sufficiently small but still $O(1)$. These restrictions on $h$ will be clear from the context. A requirement on the size of $h$ is not a limitation of the results as the interest is in the case that $h$ gets arbitrarily small.

1. The Discrete Green's Function for Half-Planes. Place a square grid over the plane with grid width $h$ such that the origin is a grid point. Let $Q=(\xi, \eta)$ be an arbitrary but fixed grid point with $\eta \geqq 0$. Let $P=(x, y)$ be any grid point with $y \geqq 0$; we denote the set of all such points by $\pi^{+}$if $y>0$ and by $\partial \pi^{+}$if $y=0$. Let $a$ and $b$ be arbitrary real numbers and let $L(a, b)$ be the discrete analogue of the logarithm function given by the relation [3, p. 422] or [7]

$$
L(a, b)=\frac{1}{2 \pi}\left\{\int_{0}^{\pi} \frac{1-\cos [b \lambda / h] \exp [-|a| \mu / h] d \lambda}{\sinh \mu}+\log h-\frac{\log 8+2 \gamma}{2}\right\}
$$

where $\cos \lambda+\cosh \mu=2$ with $\mu / \lambda \rightarrow 1$ as $\lambda \rightarrow 0$, and $\gamma$ is Euler's constant.

Let us define the function $G(P ; Q)$ by the relation

$$
G(P ; Q)=L(x-\xi, y+\eta)-L(x-\xi, y-\eta) .
$$


This mesh function is called the discrete Green's function for the upper half-plane.

We shall show in this section that if $D^{(m)} G(P ; Q)$ denotes an $m$ th order difference quotient of $G(P ; Q)$ as described in the introduction, then there exist absolute constants $A_{m}$ and $B_{m}$ such that $\rho_{P Q}^{m}\left|G^{(m)}(P ; Q)\right| \leqq A_{m}$ and $\rho_{P Q}^{m+1}\left|G^{(m)}(P ; Q)\right| \leqq B_{m} d(Q)$ where $\rho_{P Q}^{2}$ is the square of the distance from $P$ to $Q$ plus $h^{2}$ and $d(X)$ is the distance from $X$ to $\partial \pi^{+} ; A_{m}$ and $B_{m}$ are independent of $h$.

Now we will prove a collection of results which will be used frequently in deriving our estimates.

LEMMA 1.1. (a) For each mesh point $P \in \pi^{+}$, we have that

$$
\begin{aligned}
\Delta_{h} G(P ; Q)= & {[G(x+h, y ; Q)+G(x-h, y ; Q)} \\
& +G(x, y+h ; Q)+G(x, y-h ; Q)-4 G(P ; Q)] / h^{2}=-\delta(P ; Q) / h^{2}
\end{aligned}
$$

where $\delta(P ; Q)=0$ if $P \neq Q, \delta(P ; Q)=1$ if $P=Q$, and $G(P ; Q)=0$ for $P \in \partial \pi^{+}$.

(b) For all real numbers $\alpha$ and $\beta$, we have that $L(\alpha, \beta)=L(\beta, \alpha)$. In fact, $L(\alpha, \beta)$ is symmetric about the lines $\alpha=\beta, \alpha=-\beta, \alpha=0$ and $\beta=0$.

(c) For $\lambda$ and $\mu$ related as in (1), we have that $\lambda / \operatorname{sh} \mu \geqq \sin \lambda / \operatorname{sh} \mu \geqq 0$; for brevity, we use sh $X$ for $\sinh X$.

(d) If $\lambda \in(0, \pi)$, then $\lambda /(1.8) \leqq \mu \leqq \lambda$.

(e) The function $f(\mu)=(1-\exp (-a \mu)) /(\exp (2 \mu)-1)$ is positive and monotonically decreasing for $a \geqq 1$.

(f) If $0 \leqq s \leqq r$, then $\exp (-r \pm s) \mu /(1+\exp \mu)$ and $\exp (-r-1+s) \mu /(1+\exp (-\mu))$ are positive and monotone decreasing functions.

(g) The function $A(\mu)=\{\operatorname{sh}((r+1) \mu)-\operatorname{sh}(r \mu)\} \exp (-s \mu) / \operatorname{sh} \mu$ is positive and monotone decreasing for $s \geqq r+1$ with $s \geqq 0$ and $r \geqq 0$.

(h) The following elementary inequalities are true:

(i) $\mu$ ch $\mu \geqq \operatorname{sh} \mu$ for $\mu \geqq 0$;

(ii) $\sin x \leqq x$ for $x \geqq 0$;

(iii) $a+2 \leqq a \exp (-2 \mu)+2 \exp (a \mu)$ where $a \geqq 1$ and $\mu \geqq 0$;

(iv) $0 \leqq \lambda / \mathrm{sh} \mu \leqq 1.3$ for $\lambda \in[0, \pi]$ and $\cos \lambda+\operatorname{ch} \mu=2$;

(v) $x \exp (-a \pi / \alpha x) \leqq 1$ if $x \geqq 0, \alpha>0$, and $a \geqq 1$.

Proof. (a) follows closely the reasoning in [5] and (b) follows from (1).

(c) From elementary considerations,

$$
\begin{aligned}
\lambda / \operatorname{sh} \mu & \geqq \sin \lambda / \operatorname{sh} \mu=\left(\left(1-\cos ^{2} \lambda\right) /\left((2-\cos \lambda)^{2}-1\right)\right)^{1 / 2} \\
& =((1+\cos \lambda) /(3-\cos \lambda))^{1 / 2} \geqq 0 .
\end{aligned}
$$

(d) Let $g(\lambda)=\lambda-\mu$. Since $\cos \lambda+\operatorname{ch} \mu=2$ with $\mu / \lambda \rightarrow 1$ as $\lambda \rightarrow 0$, then $g(0)=0$ and $g^{\prime}(\lambda)=1-((1+\cos \lambda) /(3-\cos \lambda))^{1 / 2} \geqq 0$. Therefore, for $\lambda \in[0, \eta]$, $\lambda \geqq \mu$. Now observe that $\operatorname{sh} \mu \geqq \lambda\left(1+\lambda^{2} / 24\right)$ for $\lambda \in(0, \pi)$. Since $d^{2} \lambda / d \mu^{2}=$ $2-\cos \lambda$ ch $\mu \div \sin ^{3} \lambda \geqq 0, \max \lambda / \mu=\pi / \operatorname{ch}^{-1}(3) \leqq 1.8$.

(e) Simply observe that $f^{\prime}(\mu) \leqq 0$ since $a+2 \leqq a \exp (-2 \mu)+2 \exp (a \mu)$.

(f) Set $g(\mu)=\exp (-r-1+s) \mu /(1+\exp (-\mu))$; we see that $g^{\prime}(\mu)$

$$
=\{(-r-1+s) \exp ((-r-1+s) \mu)(1+\exp (-\mu))+\exp (-r-1+s) \mu\} /(1+\exp (-\mu))^{2} .
$$

Since $0 \leqq s \leqq r, g^{\prime}(\mu) \leqq 0$. The proof of the remaining results follows in a similar way. 
(g) Since $s \geqq r+1$, we may write $s=t+r+1$ with $t \geqq 0$. Hence, $A(\mu)=\exp (-t \mu)\{\exp \mu-\exp (-(2 r+1) \mu)+\exp (-2 r \mu)-1\} /(\exp (2 \mu)-1)$.

Our result now follows from (e) and (f).

(h) These results follow elementary considerations.

We are now ready to establish the principal result of this section. Before we proceed with this, however, we must make an obvious remark: If $P=(x, y), Q=$ $(\xi, \eta), P \neq Q$, and $d_{P Q}^{2}=(x-\xi)^{2}+(y-\eta)^{2}$, then $1 / d_{P O} \leqq \sqrt{ } 2 / \rho_{P Q}$.

THEOREM 1.1. We have the following estimates for the discrete Green's function for the upper half-plane:

(a) $|G(P ; Q)| \leqq(4.2) \sqrt{ } 2 d(Q) / \pi \rho_{P Q}$ and $|G(P ; Q)| \leqq(4.2) \sqrt{ } 2 d(P) / \pi \rho_{P Q}$.

$$
\left|G_{y}(P ; Q)\right| \leqq(4.6) d(Q) / \rho_{P Q}^{2},\left|G_{x}(P ; Q)\right| \leqq(8.6) d(Q) / \pi \rho_{P Q}^{2} \text {, }
$$

$$
\left|G_{\bar{y}}(P ; Q)\right| \leqq(11.5) d(Q) / \rho_{P Q}^{2},\left|G_{\dot{x}}(P ; Q)\right| \leqq(2.5)(8.6) d(Q) / \pi \rho_{P Q}^{2},
$$

$$
\left|G_{y}(P ; Q)\right|=\left|G_{\bar{\eta}}(P ; Q)\right|,\left|G_{x}(P ; Q)\right|=\left|G_{\bar{\xi}}(P ; Q)\right|,
$$

(c)

$$
\begin{aligned}
& \left|G_{\bar{\nu}}(P ; Q)\right|=\left|G_{\eta}(P ; Q)\right|,\left|G_{\bar{x}}(P ; Q)\right|=\left|G_{\xi}(P ; Q)\right| . \\
& \left|G_{\nu}(P ; Q)\right| \leqq(2.8) \sqrt{ } 2 / \pi \rho_{P Q},\left|G_{x}(P ; Q)\right| \leqq(2.3) \sqrt{ } 2 / \pi \rho_{P Q},
\end{aligned}
$$

$$
\left|G_{\bar{y}}(P ; Q)\right| \leqq(2.5)\left|G_{y}(P ; Q)\right|,\left|G_{\dot{x}}(P ; Q)\right| \leqq(2.5)\left|G_{x}(P ; Q)\right| \text {. }
$$

$$
\left|G_{x \dot{x}}(P ; Q)\right|=\left|G_{\xi \bar{\xi}}(P ; Q)\right|=\left|G_{x \xi}(P ; Q)\right|=\left|G_{\dot{x} \bar{\xi}}(P ; Q)\right| \leqq 14 \sqrt{ } 2 / \pi \rho_{P Q}^{2},
$$

(d) $\left|G_{y \bar{\nu}}(P ; Q)\right|=\left|G_{\eta \bar{\eta}}(P ; Q)\right|=\left|G_{\nu \bar{\eta}}(P ; Q)\right|=\left|G_{\bar{\nu} \hat{\eta}}(P ; Q)\right| \leqq 21 / \pi \rho_{P Q}^{2}$,

$$
\left|G_{x y}(P ; Q)\right|=\left|G_{x \bar{\eta}}(P ; Q)\right|=\left|G_{\bar{\xi}_{\nu}}(P ; Q)\right|=\left|G_{\bar{\xi} \bar{\eta}}(P ; Q)\right| \leqq(6.9) / \pi \rho_{P Q}^{2} .
$$

$$
\left|G_{x y}(P ; Q)\right| \leqq(10.9) d(Q) / \rho_{P Q}^{3},\left|G_{x \bar{x}}(P ; Q)\right| \leqq(21.2) d(Q) / \rho_{P Q}^{3}
$$

$$
\left|G_{\nu \bar{y}}(P ; Q)\right| \leqq(21.2) d(Q) / \rho_{P Q}^{3} .
$$

Proof. (a) Let $r=y / h, s=\eta / h$ and $t=|x-\xi| / h$. Then

$$
\begin{aligned}
|G(P ; Q)| & =\frac{1}{\pi}\left|\int_{0}^{\pi} \frac{\sin (r \lambda) \sin (s \lambda) \exp (-t \mu)}{\operatorname{sh} \mu} d \lambda\right| \leqq \frac{1}{\pi} \int_{0}^{\pi} \frac{r \lambda \exp (-t \mu)}{\operatorname{sh} \mu} d \lambda \\
& \leqq \frac{(1.3) r}{\pi} \int_{0}^{\pi} \exp (-t \mu) d \lambda \\
& \leqq \frac{(1.3) r}{\pi} \int_{0}^{\pi} \exp (-t \lambda) /(1.8) d \lambda \leqq(2.4) y / \pi|x-\xi|
\end{aligned}
$$

here we have used (c) and (d) of Lemma 1.1. By a similar line of reasoning, we may conclude that $|G(P ; Q)| \leqq(2.4) \eta / \pi|x-\xi|$.

Now we write, using the symmetry of $L(\alpha, \beta)$,

$$
\begin{array}{rlrl}
G(P ; Q) & =\frac{1}{2 \pi} \int_{0}^{\pi} \frac{\cos (t \lambda) \exp ([-s+r] \mu)(1-\exp (-2 r \mu))}{\operatorname{sh} \mu} d \lambda, & & \text { if } r \leqq s, \\
& =\frac{1}{2 \pi} \int_{0}^{\pi} \frac{\cos (t \lambda) \exp ([-r+s] \mu)(1-\exp (-2 s \mu))}{\operatorname{sh} \mu} d \lambda, & \text { if } r \geqq s .
\end{array}
$$

For $r \leqq s$, we have 


$$
\begin{aligned}
\mid \text { r.h.s. (3.1)| } & \leqq\left|\frac{1}{2 \pi} \int_{0}^{\pi} \frac{2 \exp [(r-s) \mu] r \mu}{\operatorname{sh} \mu} d \lambda\right| \\
& \leqq\left|\frac{1}{\pi} \int_{0}^{\pi} r \exp [(r-s) \mu] d \lambda\right| \leqq(1.8) r / \pi|r-s| .
\end{aligned}
$$

In a similar way, $\mid$ r.h.s.(3.2) $|\leqq(1.8) s / \pi| r-s \mid$ for $r \geqq s$. Hence, we have $|G(P ; Q)| \leqq$ (1.8) $\eta / \pi|y-\eta|$ and $|G(P ; Q)| \leqq(1.8) y / \pi|y-\eta|$.

Combining the results of these two paragraphs yields the estimate, for $P \neq Q$,

$$
\rho_{P O}|G(P ; Q)| \leqq(|x-\xi|+|y-\eta|)|G(P ; Q)| \leqq\left\{\begin{array}{l}
(4.2) \eta / \pi \\
\text { and } \\
(4.2) y / \pi
\end{array}\right.
$$

If $P=Q,|G(Q ; Q)| \leqq(1.3) y / h$ or $|G(Q ; Q)| \leqq(1.3) \eta / h$.

(b) We first consider

$$
\begin{aligned}
G_{y}(P ; Q)= & {[G(x, y+h ; \xi, \eta)-G(x, y ; \xi, \eta)] / h } \\
= & {[L(\xi-x,-\eta-y-h)-L(\xi-x, \eta-y-h)} \\
& \quad+L(\xi-x,-\eta-y)-L(\xi-x, \eta-y)] / h \\
= & {[L(t,-s-r-1)-L(t, s-r-1)-L(t,-s-r)+L(t, s-r)] / h } \\
= & \frac{1}{2 \pi h} 2 \int_{0}^{\pi} \cos (t \lambda) A(\mu) d \lambda, \quad \text { for } s \geqq r+1, \\
(4) \quad & \frac{1}{2 \pi h} 2 \int_{0}^{\pi} \cos (t \lambda) B(\mu) d \lambda, \quad \text { for } s \leqq r,
\end{aligned}
$$

where $A(\mu)$ is given in Lemma $1.1(\mathrm{~g})$ and

$$
B(\mu)=\exp (-r \mu)[\exp (-\mu)-1] \operatorname{sh}(s \mu) / \operatorname{sh} \mu .
$$

Since $A(\mu)$ is monotone decreasing in $\mu$ and since, by Lemma $1.1(\mathrm{c}), d \mu / d \lambda=$ $\sin \lambda / \operatorname{sh} \mu \geqq 0$, we have that $A(\mu)$ is monotone decreasing in $\lambda$; let $\tilde{A}(\lambda)$ denote $A(\mu)$ as a function of $\lambda$. Looking at (4.1), we write

$$
\int_{0}^{\pi} \tilde{A}(\lambda) \cos (t \lambda) d \lambda=\frac{1}{t} \int_{0}^{\pi t} \tilde{A}(\lambda) \cos z d z
$$

Decompose the interval $[0, t \pi]$, for $t \neq 0$, into $\{[0, \pi / 2],[\pi / 2,3 \pi / 2], \cdots,[(2 k+1) \pi / 2$, $t \pi]\}$ or into $\{[0, \pi / 2],[\pi / 2,3 \pi / 2], \cdots,[(2 k-1) \pi / 2,(2 k+1) \pi / 2]\}$ where, in the latter case, we have that $(2 k+1) \pi=2 t \pi$. Observing the alteration in the signum of the integrand over each interval in either decomposition of $[0, t \pi]$, we conclude that (here we are using the estimates $r-s \leqq-1$, and $t \exp ((r-s) \pi / 6 t) \leqq 1$; we are assuming $t \neq 0$ and $\eta \geqq h$ )

$$
\begin{aligned}
\mid \int_{0}^{\pi} A(\mu) & \cos (t \lambda) d \lambda \mid \leqq 2 \int_{0}^{(\pi / 2) t} A(\mu) \cos (t \lambda) d \lambda \\
& \leqq\left.(1.8)^{2} \exp [(r-s) \lambda / 1.8]\{((r-s) / 1.8) \cos (t \lambda)+t \sin ((r-s) \lambda / 1.8)\}\right|_{0} ^{\pi / 2 t} \\
& \leqq(3.6)[s-r+1.8] /\left((r-s)^{2}+t^{2}\right) .
\end{aligned}
$$


Therefore,

$$
\left|G_{y}(P ; Q)\right| \leqq(4.6) d(Q) / \rho_{P Q}^{2} \quad \text { if } \eta \geqq y+h .
$$

The case $t=0$ is treated in a similar manner and we obtain the estimate, assuming $\eta \geqq y+h$ or $y=\eta$ or $\eta=0$ or $x=\xi$,

$$
\left|G_{\nu}(P ; Q)\right| \leqq(3.6) \sqrt{ } 2 d(Q) / \pi \rho_{P Q}^{2} .
$$

Now we consider the integral in (4.2). By elementary considerations, we obtain the identity

$$
\begin{aligned}
\int_{0}^{\pi} B(\mu) \cos (t \lambda) d \lambda= & -\int_{0}^{\pi} \cos (t \lambda) \exp [(s-r) \mu] d \lambda /(1+\exp \mu) \\
& +\int_{0}^{\pi} \cos (t \lambda) \exp [(-s-r) \mu] d \lambda /(1+\exp \mu)
\end{aligned}
$$

and the inequality

$$
\begin{aligned}
\left|\int_{0}^{\pi} B(\mu) \cos (t \lambda) d \lambda\right| \leqq & \int_{0}^{(\pi / 2) t} \cos (t \lambda) \exp [(s-r) \mu] d \lambda /(1+\exp \mu) \\
& +\int_{0}^{\pi / 2 t} \cos (t \lambda) \exp [-(r+s) \mu] d \lambda /(1+\exp \mu)
\end{aligned}
$$

Hence, if $r \neq s$,

$$
\begin{aligned}
\mid \int_{0}^{\pi} B(\mu) & \cos (t \lambda) d \lambda \mid \\
& =\left|\int_{0}^{\pi} \cos (t \lambda)\{\exp [(s-r) \mu]-\exp [(-s-r) \mu]\} d \lambda /(1+\exp \mu)\right| \\
& \leqq \frac{1}{2}\left|\int_{0}^{\pi}\{\exp [(s-r) \mu]-\exp [(-s-r) \mu]\} d \lambda\right| \\
& \leqq(1.3)\left[2 s / r^{2} s^{2}\right] / 2 \leqq(1.3)\left[s /(r-s)^{2}\right]
\end{aligned}
$$

If $r=s(s \geqq 1$ as we have already treated the case $\eta=0)$, then

$$
\begin{aligned}
\left|\int_{0}^{\pi} B(\mu) \cos (t \lambda) d \lambda\right| & =\left|\int_{0}^{\pi} \cos (t \lambda)[1-\exp [-2 s \mu]] d \lambda /(1-\exp [-2 \mu])\right| \\
& =\left|\int_{0}^{\pi} \cos (t \lambda) \sum_{k=0}^{s-1} \exp [-s k \mu] \cdot \exp [-\mu] d \lambda\right| \\
& \leqq\left|s \int_{0}^{(\pi / 2) t} \cos (t \lambda) \exp [-\mu] d \lambda\right| \leqq 2 s /\left(1+t^{2}\right)
\end{aligned}
$$

Therefore, when $r=s$,

$$
\left|G_{y}(P ; Q)\right| \leqq \eta / \pi(x-\xi)^{2} .
$$

Combining the inequalities in (5), (6), (7) and (8), we have

$$
\left|G_{\nu}(P ; Q)\right| \leqq(4.6) d(Q) / \rho_{P Q}^{2} .
$$

Now we turn our attention to an estimate of the term 


$$
\begin{aligned}
G_{x}(P ; Q)= & \{G(x+h, y ; \xi, \eta)-G(x, y ; \xi, \eta)\} / h \\
= & \{L(x+h-\xi, y+\eta)-L(x+h-\xi, y-\eta) \\
& -L(x-\xi, y+\eta)+L(x-\xi, y-\eta)\} / h .
\end{aligned}
$$

Elementary considerations give

$$
\begin{aligned}
G_{x}(P ; Q) & =\frac{1}{\pi h} \int_{0}^{\pi} \frac{\sin (r \lambda) \sin (s \lambda)(\exp (-t-1) \mu-\exp (-t \mu))}{\operatorname{sh} \mu} d \lambda, & & \text { if } t \geqq 1, \\
& =\frac{1}{\pi h} \int_{0}^{\pi} \frac{\sin (r \lambda) \sin (s \lambda)\left(\exp \left(-t^{\prime}+1\right) \mu-\exp \left(-t^{\prime} \mu\right)\right)}{\operatorname{sh} \mu} d \lambda, & & \text { if } t \leqq 0,
\end{aligned}
$$

where $t^{\prime}=(\xi-x) / h, t=(x-\xi) / h$ and $r, s$ are as defined in the proof of part (a). Therefore,

$$
\begin{aligned}
\left|G_{x}(P ; Q)\right| & \leqq \frac{1}{\pi h} \int_{0}^{\pi} \frac{s \lambda \exp (-t \mu)(1-\exp (-\mu))}{\operatorname{sh} \mu} d \lambda, \quad \text { if } t \geqq 1, \\
& \leqq \frac{1}{\pi h} \int_{0}^{\pi} \frac{s \lambda \exp \left(-t^{\prime} \mu\right)(1-\exp (-\mu))}{\operatorname{sh} \mu} d \lambda, \quad \text { if } t \leqq 0 .
\end{aligned}
$$

Hence,

$$
\left|G_{x}(P ; Q)\right| \leqq(1.8)^{2}(1.3) \eta / \pi(x-\xi)^{2} .
$$

When $t=0$, we easily obtain $\left|G_{x}(P ; Q)\right| \leqq \pi \eta / 2 h^{2}$.

Using the symmetry of $L(r, t)$, we may write

$$
\begin{aligned}
G_{x}(P ; Q)=\{L(y+\eta, x+h-\xi) & -L(y-\eta, x+h-\xi) \\
& -L(y+\eta, x-\xi)+L(x-\xi, y-\eta)\} / h \\
=\frac{1}{2 \pi h} \int_{0}^{\pi}(\cos (t+1) \lambda- & \cos (t \lambda)) \\
\cdot & \{\exp (-|r-s| \mu)-\exp (-|r+s| \mu)\} d \lambda / \operatorname{sh} \mu .
\end{aligned}
$$

Hence,

$$
\begin{aligned}
\left|G_{x}(P ; Q)\right| & \leqq \frac{(1.3)}{h \pi} \int_{0}^{\pi} s \mu \exp (s-r) \mu d \lambda \quad \text { for } r \geqq s, \\
& \leqq \frac{(1.3)}{h \pi} \int_{0}^{\pi} r \mu \exp (r-s) \mu d \lambda \quad \text { for } r \leqq s .
\end{aligned}
$$

Elementary considerations applied to this estimate yield

$$
\left|G_{x}(P ; Q)\right| \leqq(4.3) \eta / \pi(y-\eta)^{2} .
$$

Applying (10) and (12) to the expression $\left\{(x-\xi)^{2}+(y-\eta)^{2}\right\}\left|G_{x}(P ; Q)\right|$ gives

$$
\left|G_{x}(P ; Q)\right| \leqq(8.6) \eta / \pi \rho_{P Q}^{2} .
$$

The estimates for $G_{\dot{\nu}}(P ; Q)$ and $G_{\dot{x}}(P ; Q)$ can be derived, as we did for $G_{\nu}(P ; Q)$ and $G_{x}(P ; Q)$, or, we may simply make the following observations: If $P^{\prime}=(x, y-h)$, then $\left|G_{\dot{\nu}}(P ; Q)\right|=|G(x, y ; Q)-G(x, y-h ; Q)| / h=\left|G_{\nu}\left(P^{\prime} ; Q\right)\right| \leqq(4.6) d(Q) / \rho_{P^{\prime},}^{2}$. 
But $d(Q) / \rho_{P^{\prime} O}^{2} \leqq(2.5) d(Q) / \rho_{P Q}^{2}$. Hence, $\left|G_{\dot{\nu}}(P ; Q)\right| \leqq(2.5)(4.6) d(Q) \rho_{P Q}^{2}$. A similar analysis applies to $G_{\dot{x}}(P ; Q)$.

The remaining results of (b) follow easily. For example,

$$
\begin{aligned}
\left|G_{y}(P ; Q)\right|= & (1 / h)\left|L_{y}(x, y ; \xi,-\eta)-L_{y}(x, y ; \xi, \eta)\right| \\
= & \mid L(x-\xi, y+h+\eta)-L(x-\xi, y+\eta) \\
& -L(x-\xi, y+h-\eta)+L(x-\xi, y-\eta) \mid / h \\
= & \left|G_{\tilde{\eta}}(P ; Q)\right|=\left|L_{\tilde{\eta}}(x, y ; \xi,-\eta)-L_{\tilde{\eta}}(x, y ; \xi, \eta)\right| / h \\
= & \mid L(x-\xi, y+\eta)-L(x-\xi, y+\eta+h) \\
& -L(x-\xi, y-\eta)+L(x-\xi, y-\eta+h) \mid / h .
\end{aligned}
$$

Note here that care must be exercised when $Q$ is near the boundary. For example, if $Q=(\xi, 0)$, then, clearly, $\left|G_{i j}(P ; Q)\right|=\left|G_{\eta}(P ; Q)\right|$ is not a meaningful relation, in that $G_{i j}(P ; Q)$ is not defined for $Q$ on the boundary. In such a case, we note facts of the kind that $\left|G_{\eta}(P ; \xi, 0)\right|=\left|G_{\dot{\nu}}(P ; \xi, h)\right|$ from which a proper inequality can be drawn.

(c) For both cases in (4), we have that

$$
\left|G_{\nu}(P ; Q)\right| \leqq \int_{0}^{(\pi / 2) t} \cos (t \lambda) d t=1 / \pi h t
$$

as $\exp (-s \mu)\{\operatorname{sh}(r+1) \mu-\operatorname{sh}(r \mu)\} / \operatorname{sh} \mu \leqq \mu \exp (-s \mu) \operatorname{ch}(r+1) \mu / \operatorname{sh} \mu \leqq 1$ and $\exp (-r \mu) \operatorname{sh}(s \mu)(\exp (-\mu)-1) / \operatorname{sh} \mu \leqq 1$. Therefore,

$$
\left|G_{y}(P ; Q)\right| \leqq 1 / \pi|x-\xi| \text { for } t \neq 0, \quad\left|G_{y}(P ; Q)\right| \leqq 1 / h \quad \text { for } t=0 .
$$

Now observe that

$$
\begin{aligned}
|(4.1)| & \leqq \frac{1}{\pi h} \int_{0}^{\pi} \frac{\exp (-s \mu)[\operatorname{sh}(r+1) \mu-\operatorname{sh}(r \mu)]}{\operatorname{sh} \mu} d \lambda \\
& \leqq \frac{1}{\pi h} \int_{0}^{\pi} \exp ((r-s) \mu)[1+\exp (-2 r \mu)] d \lambda \\
& \leqq \frac{2}{\pi h} \int_{0}^{\pi} \exp ((r-s) \lambda / 1.8) d \lambda \leqq 3.6 / \pi|y-\eta|
\end{aligned}
$$

and

$$
|(4.2)| \leqq \frac{1}{\pi h} \int_{0}^{\pi} \frac{\exp (-r \mu)[\operatorname{sh}(s \mu)(\exp (-\mu)-1)]}{\operatorname{sh} \mu} d \lambda \leqq 1.8 / \pi|y-\eta| .
$$

Combining (13) and (14) and using the inequality $d_{P Q} \leqq|y-\eta|+|x-\eta|$ gives

$$
\left|G_{\nu}(P ; Q)\right| \leqq(2.8) \sqrt{ } 2 / \pi \rho_{P O} .
$$

We may also write, using (9) and the fact that $|\sin x| \leqq 1$,

$$
\begin{aligned}
& \left|G_{x}(P ; Q)\right| \leqq \frac{1}{\pi h} \int_{0}^{\pi} \exp (-t \mu) d \lambda, \quad x+h \geqq \xi, \\
& \leqq \frac{1}{\pi h} \int_{0}^{\pi} \exp \left(-t^{\prime} \mu\right) d \lambda, \quad x \leqq \xi .
\end{aligned}
$$


Hence,

$$
\left|G_{x}(P ; Q)\right| \leqq 1.8 / \pi|x-\xi|
$$

If $t=0,\left|G_{x}(P ; Q)\right| \leqq 1 / h$. Also, using the symmetry of $L(\alpha, \beta)$,

$\left|G_{x}(P ; Q)\right|=\frac{1}{2 \pi h} \int_{0}^{\pi} \frac{[\cos (t+1) \lambda-\cos t \lambda][\exp (-|r-s| \mu)-\exp (-|r+s| \mu)]}{\operatorname{sh} \mu} d \lambda$

$$
\leqq \frac{1.8}{2 \pi h} \int_{0}^{\pi} \exp ((s-r) \mu)(1-\exp (-2 s \mu)) d \lambda, \quad r \geqq s,
$$

$$
\begin{aligned}
& \leqq \frac{1.8}{2 \pi h} \int_{0}^{\pi} \exp ((r-s) \mu)(1-\exp (-2 r \mu)) d \lambda, \quad r \leqq s, \\
& \leqq 1.17 \pi /|\eta-y| .
\end{aligned}
$$

If $y=\eta,\left|G_{x}(P ; Q)\right| \leqq .9 / h$. From (15) and (16), we have

$$
\left|G_{x}(P ; Q)\right| \leqq(2.97) \sqrt{ } 2 / \pi \rho_{P Q} .
$$

(d) We have that

$$
\begin{aligned}
G_{x \dot{x}}(P ; Q)= & \{G(x+h, y ; \xi, \eta)+G(x-h, y ; \xi, \eta)-2 G(x, y ; \xi, \eta)\} / h^{2} \\
= & \{L(x+h-\xi, y+\eta)-L(x+h-\xi, y-\eta)+L(x-h-\xi, y+h) \\
& \quad-L(x-h-\xi, y-\eta)-2(L(x-\xi, y+\eta)-L(x-\xi, y-\eta))\} / h^{2} \\
(17) \quad= & \frac{1}{2 \pi h^{2}} \int_{0}^{\pi}[\cos (r-s) \lambda-\cos (r+s) \lambda] \\
& \quad \cdot[\exp (-|t+1| \mu)+\exp (-|t-1| \mu)-2 \exp (-|t| \mu)] d \lambda / \operatorname{sh} \mu \\
= & \frac{1}{2 \pi h^{2}} \int_{0}^{\pi}[\exp (-|r-s| \mu)-\exp (-|r+s| \mu)] \\
& \cdot[\cos (t+1) \lambda+\cos (t-1) \lambda-2 \cos (t \lambda)] d \lambda / \operatorname{sh} \mu .
\end{aligned}
$$

For the first integral in (17), we have

$$
\begin{aligned}
& \left|G_{x \dot{x}}(P ; Q)\right| \leqq \frac{1}{\pi h^{2}} \int_{0}^{\pi}|\exp (-t \mu)[\exp (-\mu)+\exp \mu-2] d \lambda / \operatorname{sh} \mu|, \quad x \geqq \xi, \\
& \leqq \frac{1}{\pi h^{2}} \int_{0}^{\pi}|\exp (t \mu)[\exp \mu+\exp (-\mu)-2] d \lambda / \operatorname{sh} \mu|, \quad x \leqq \xi .
\end{aligned}
$$

Now $\exp (-\mu)+\exp \mu-2=2$ [ch $\mu-1$ ]. Taking the derivative with respect to $\mu$, using the equality ch $\mu=2-\cos \lambda$ and the estimate $\operatorname{sh} \mu \leqq \mu$ ch $\mu$ gives the inequality $2[\operatorname{ch} \mu-1] \leqq 3 \mu^{2}$ when $\lambda \in[0, \pi]$. Applying this result to (18) gives the estimate

$$
\left|G_{x x}(P ; Q)\right| \leqq \frac{3}{\pi h^{2}}\left\{\begin{array}{ll}
\int_{0}^{\pi} \exp (-t \mu) \mu d \lambda, & x \geqq \xi \\
\int_{0}^{\pi} \exp (t \mu) \mu d \lambda, & x \leqq \xi
\end{array}\right\} \leqq 3(1.8)^{2} / \pi(x-\xi)^{2} .
$$

For the second integral in (17), we have 


$$
\begin{aligned}
\left|G_{x \dot{x}}(P ; Q)\right| & \leqq \frac{1}{\pi h^{2}} \int_{0}^{\pi} \frac{\lambda^{2}[\exp (-|r-s| \mu)-\exp (-|r+s| \mu)]}{\operatorname{sh} \mu} d \lambda \\
& \leqq \frac{1.3}{\pi h^{2}} \int_{0}^{\pi} \lambda[\exp (-|r-s| \mu)-\exp (-|r+s| \mu)] d \lambda \\
& \leqq(1.3)(1.8)^{2} / \pi(y-\eta)^{2} .
\end{aligned}
$$

Combining (19) and (20) gives the estimate $\left|G_{x \dot{x}}(P ; Q)\right| \leqq 14 \sqrt{ } 2 / \pi \rho_{P Q}^{2}$. From the equation $\Delta_{h} G(P ; Q)=-\delta(P ; Q) / h^{2}$, we obtain the estimate

$$
\left|G_{\nu \hat{\nu}}(P ; Q)\right|<21 / \pi \rho_{P Q}^{2} \text {. }
$$

Using (9), we may write

$\left|G_{x y}(P ; Q)\right| \equiv\left|G_{x}(x, y+h ; Q)-G_{x}(P ; Q)\right| / h$

$$
\begin{aligned}
& =\frac{1}{\pi h^{2}}\left|\int_{0}^{\pi} \frac{(\sin (r+1) \lambda-\sin (r \lambda)) \sin (s \lambda)(\exp (-t-1) \mu-\exp (-t \mu))}{\operatorname{sh} \mu} d \lambda\right|, t \geqq 1, \\
& =\frac{1}{\pi h^{2}}\left|\int_{0}^{\pi} \frac{(\sin (r+1) \lambda-\sin (r \lambda)) \sin (s \lambda)\left(\exp \left(-t^{\prime}-1\right) \mu-\exp \left(-t^{\prime} \mu\right)\right)}{\operatorname{sh} \mu} d \lambda\right|, t \leqq 0, \\
& \leqq(1.8)^{2} / \pi(x-\xi)^{2} .
\end{aligned}
$$

From the results preceding (11), we may write

$\left|G_{x y}(P ; Q)\right|=\frac{1}{2 \pi h^{2}} \mid \int_{0}^{\pi}(\cos (t+1) \lambda-\cos (t \lambda))$

$$
\begin{aligned}
& \cdot\{\exp (-|r+1-s| \mu)-\exp (-|r-s| \mu) \\
&+\exp (-|r+s+1| \mu)-\exp (-|r+s| \mu)\} d \lambda / \operatorname{sh} \mu \mid \\
& \leqq(1.62) / \pi(y-\eta)^{2} ;
\end{aligned}
$$

we note here that $|\exp (-|r-s+1| \mu)-\exp (-|r-s| \mu)| \leqq \exp (-|r-s| \mu)(\exp \mu-1)$, $\cos ((t+1) \lambda)-\cos (t \lambda)=\sin [(t+\epsilon) \lambda] \lambda$ with $0 \leqq \epsilon \leqq 1$, and $\exp (-|r+s+1| \mu)$ $-\exp (-|r+s| \mu)<0$. Hence,

$$
\left|G_{x y}(P ; Q)\right| \leqq(6.9) / \pi \rho_{P Q}^{2} .
$$

(e) From (21), we have

$$
\left|G_{x y}(P ; Q)\right| \leqq \frac{s}{\pi h^{2}} \int_{0}^{\pi} \lambda^{2} \exp (-t \mu) d \lambda \leqq(11.7) \eta / \pi|x-\xi|^{3} .
$$

Using (22), we obtain the estimate

$$
\begin{aligned}
\left|G_{x y}(P ; Q)\right| & \leqq \frac{s}{\pi h^{2}} \int_{0}^{\pi} \lambda \mu \exp ((-r+s) \mu) d \lambda, & & r \geqq s, \\
& \leqq \frac{r}{\pi h^{2}} \int_{0}^{\pi} \lambda \mu \exp ((-r+s) \mu) d \lambda, & & r<s .
\end{aligned}
$$

Hence we have that $\left|G_{x y}(P ; Q)\right| \leqq(11.7) \eta / \pi|y-\eta|^{3}$. Combining this with (23) gives our estimate.

From the first integral in (17), we obtain the estimate 


$$
\begin{array}{rlrl}
\left|G_{x \dot{x}}(P ; Q)\right| & \leqq \frac{3}{\pi h^{2}} \int_{0}^{\pi} \frac{\eta \lambda \mu^{2} \exp (-t \mu)}{\operatorname{sh} \mu} d \lambda, & & x \geqq \xi, \\
& \leqq \frac{3}{\pi h^{2}} \int_{0}^{\pi} \frac{\eta \lambda \mu^{2} \exp \left(-t^{\prime} \mu\right)}{\operatorname{sh} \mu} d \lambda, & x \leqq \xi .
\end{array}
$$

Therefore, $\left|G_{x \dot{x}}(P ; Q)\right|=\left|G_{y \hat{y}}(P ; Q)\right| \leqq 6(1.8)^{3} \eta / \pi|x-\xi|^{3}$ for $P \neq Q$.

The second integral in (17) allows us to get the estimate

$$
\begin{aligned}
\left|G_{x \bar{x}}(P ; Q)\right| & \leqq \frac{1}{\pi h^{2}} \int_{0}^{\pi} \frac{\eta \mu \lambda^{2} \exp ((-r+s) \mu)}{\operatorname{sh} \mu} d \lambda, & & r \geqq s, \\
& \leqq \frac{1}{\pi h^{2}} \int_{0}^{\pi} \eta \mu \lambda^{2} \exp ((-s+r) \mu) d \lambda, & & r<s,
\end{aligned}
$$

Hence, $\left|G_{x x}(P ; Q)\right| \leqq 2(1.8)^{3} \eta / \pi|y-\eta|^{3}$. Our proof is now complete.

By the methods presented in the proof of the last theorem, we may prove the following result: If $m$ is any integer, then constants $B_{m}$ and $C_{m}$ exist, depending only on $m$, such that

$$
\left|D^{m} G(P ; Q)\right| \leqq B_{m} / \rho_{P Q}^{m} \text { and }\left|\tilde{D}^{m} G(P ; Q)\right| \leqq \eta C_{m} / \rho_{P Q}^{m+1}
$$

where $\tilde{D}_{m} G(P ; Q)$ is any $m$ th order difference quotient taken with respect to the components of $P$. If the difference quotient is with respect to $Q$, we have $\left|\tilde{D}^{m} G(P ; Q)\right| \leqq$ $y D_{m} / \rho_{P Q}^{m+1}$.

Having examined the discrete Green's function for the upper half-plane, we may now observe that the same estimates hold for the lower half-plane. If we consider the discrete Green's function for the right half-plane or the left half-plane, then the same estimates of Theorem 1.1 hold except that we replace the quantity $\eta$ by $\xi$ in parts (b) and (c) of that theorem.

For the mesh region described, let us look at the line $y=-x$. This intersects grid points at a spacing of $\sqrt{ } 2 h$. Let $G(P ; Q)$ be the discrete Green's function for the region to the right of this line, i.e., mesh points $P=(x, y)$ such that $y>-x$. Let $Q=(\xi, \eta)$ be a mesh point in this half-plane. Let $Q^{\prime}$ be the reflection of this point about the line $y=-x$; i.e., $Q^{\prime}=(-\eta,-\xi)$. Then we have that

$$
\begin{aligned}
G(P ; Q)= & L(x-\xi, y-\eta)-L(x+\eta, y+\xi) \\
= & \int_{0}^{\pi} \exp (-|t| \mu)[\cos ((y+\xi) \lambda / h)-\cos ((r-s) \lambda)] \\
& \quad+\cos ((y+\xi) \lambda / h)[\exp (-|x+\eta| \mu / h)-\exp (-|t| \mu)] d \lambda / \operatorname{sh} \mu .
\end{aligned}
$$

As these integrals are similar to those already estimated, we may state the next theorem.

THEOREM 1.2. If $m$ is a nonnegative integer and if $G(P ; Q)$ is the discrete Green's function for the mesh region to the right or to the left of the line $y=x$ or $y=-x$, then there exist absolute constants $D_{m}$ and $E_{m}$ such that

$$
\left|D^{(m)} G(P ; Q)\right| \leqq D_{m} / \rho_{P Q}^{m}
$$

and

$$
\left|\tilde{D}^{(m)} G(P ; Q)\right| \leqq E_{m}|(\xi+\eta) / 2| / \rho_{P Q}^{m+1}
$$


2. Some Other Infinite Regions. Let $S$ be the strip bounded by the lines $y=0$ and $y=a$; we assume with no loss of generality that $a / h$ is an integer. Let $G^{S}(P ; Q)$ be the discrete Green's function associated with the operator $\Delta_{h}$ and the set $S_{h} ; S_{h}$ is the set of grid points in the interior of $S$. We have the following result.

THEOREM 2.1. If $G^{H}\left(P ; Q_{i}\right)$ is the discrete Green's function for the upper halfplane with singularity at $Q_{i}=\left(\xi, \eta_{j}\right)$, then

$$
G^{S}(P ; Q)=\sum_{i=0}^{\infty}(-1)^{i} G^{H}\left(P ; Q_{i}\right),
$$

where $Q=Q_{0}=(\xi, \eta), Q_{1}=(\xi, 2 a-\eta), Q_{2}=(\xi, 2 a+\eta), Q_{3}=(\xi, 4 a-\eta)$, $Q_{4}=(\xi, 4 a+\eta), \cdots, Q_{2 i}=(\xi, 2 j a+\eta), Q_{2 i+1}=(\xi, 2(j+1) a-\eta), \cdots$. that

In fact, there exist absolute constants $H_{m}, J_{m}$ and $M$, each independent of $h$, such

$$
\begin{array}{ll}
\left|\tilde{D}^{(m)} G^{S}(P ; Q)\right| \leqq H_{m} d(Q) / \rho_{P Q}^{m+1}, & m=1,2,3, \cdots, \\
\left|D^{(m)} G^{S}(P ; Q)\right| \leqq J_{m} / \rho_{P Q}^{m}, & m=1,2, \cdots,
\end{array}
$$

and

$$
0 \leqq G^{S}(P ; Q) \leqq M \min (d(P), d(Q)) / \rho_{P Q},
$$

where $d(X)$ is the distance of $X$ to $\partial S_{h}$.

Proof. We first establish the convergence of (1). If $|x-\xi| h^{-1}=t, S_{i}=\eta_{i} / h$, $y / h=r$, then we may write $(1)$ as $G^{S}(P ; Q)=G^{H}(P ; Q)+\sum_{i=1}^{\infty}(-1)^{i} G^{H}\left(P ; Q_{i}\right)$. But

$$
\sum_{i=1}^{\infty}(-1)^{i} G^{H}\left(P ; Q_{i}\right)=2 \sum_{i=1}^{\infty}(-1)^{j} \int_{0}^{\pi}\left\{\cos (t \lambda) \exp \left[\left(-S_{i}+r-1\right) \mu\right] \sum_{K=0}^{r-1} e^{-2 K_{\mu}}\right\} d \lambda
$$

where $S_{i}$ satisfies these relations by virtue of the definition of the $\eta_{j}$.

Now we will show that, for any $K$, the series

$$
\sum_{i=1}^{\infty}(-1)^{i} \int_{0}^{\pi} \cos (\lambda t) \exp \left[\left(-S_{i}+r-1-2 K\right) \mu\right] d \lambda
$$

is convergent; hence, any finite sum of such series is convergent. Let $\mathfrak{K}\left(t, \lambda, \mu, S_{i}, K\right)$ be the integrand of the series above. Let $a_{i}=\int_{0}^{\epsilon} \mathcal{K} d \lambda$ and $b_{i}=\int_{\epsilon}^{\pi} \mathcal{K} d \lambda$ where $\epsilon \in(0, \pi / 4 t]$. Then we will show that

$$
Z_{1}(K)=\sum_{i=1}^{\infty}(-1)^{i} a_{i} \text { and } Z_{2}(K)=\sum_{i=1}^{\infty}(-1)^{i} b_{j}
$$

is convergent.

We have that $a_{i}-a_{i+1} \geqq 0$ as $S_{i}<S_{i+1}$. Also,

$$
\lim _{i \rightarrow \infty} a_{i} \leqq \lim _{i \rightarrow \infty} \int_{0}^{e} \exp \left[\left(-S_{i}+r-1-2 K\right) \lambda / 3\right] d \lambda=0 .
$$

Therefore, $Z_{1}(K)$ converges.

Now we have

$$
Z_{2}(K)=\sum_{i=1}^{\infty}(-1)^{i} \int_{\epsilon}^{\pi} f_{i}(\lambda) d \lambda=\int_{\pi / 4 t}^{\pi}\left[\sum_{i=1}^{\infty}(-1)^{i} f_{i}(\lambda)\right] d \lambda
$$

with $f_{i} \equiv \cos (t \lambda) \exp \left[\left(-S_{i}-1+r-2 K\right) \mu\right]$. The family $\left\{f_{i}(\lambda)\right\}$ is a uniformly 
convergent sequence of continuous functions for $\lambda \in[\epsilon, \pi]$. In fact,

$$
\left|f_{i}(\lambda)\right| \leqq \exp \left[\left(-S_{i}-1+r-2 K\right) \mu\right] \leqq \exp \left[\left(-S_{i}-1+r-2 K\right) \mu(\epsilon)\right]
$$

with $\mu(\epsilon)>0$ the value of $\mu$ at $\lambda=\epsilon$. Hence, $Z_{2}(K)$ converges and we may write

$$
Z_{2}(K)=\int_{\epsilon}^{\pi}\left\{\cos (t \lambda) \exp [(r-1-2 K) \mu] \sum_{i=0}^{\infty}(-1)^{i} e^{-S_{j} \mu}\right\} d \lambda .
$$

Therefore, (1) is well defined and we have

$$
G^{S}(P ; Q)=\sum_{K=0}^{r-1} Z_{2}(K)+c \epsilon
$$

where $\epsilon>0$ and $0 \leqq c \leqq 1$; this last term is due to the fact that $Z_{1}(K) \leqq \epsilon$.

Now we show that $G^{S}(P ; Q)=0$ for $P \in \partial S_{h}$. This is clear when $y=0$. Now we look at the case that $P$ is on $y=a$. Let $\delta_{N}$ be the $N$ th partial sum of $G^{S}(P ; Q)$. Rearranging the entries, we have

$$
\begin{aligned}
S_{N}=\left\{G^{\prime}\left(P ; Q_{0}\right)-G^{\prime}\left(P ; Q_{0}^{\prime}\right)+\cdots+(-1)^{N+1} G^{\prime}\left(P ; Q_{N-2}^{\prime}\right)\right. \\
\left.+(-1)^{N+1}\left[L\left(P ; Q_{N-1}^{\prime}\right)-L\left(P ; Q_{N}^{\prime}\right)\right]\right\}
\end{aligned}
$$

where $Q_{i}^{\prime}=\left(\xi,-\eta_{i}\right)$ and $G^{\prime}\left(P ; Q_{j}^{\prime}\right)$ is the discrete Green's function for the lower half-plane with boundary $y=a$. Now, along $y=a$, we have that $\left|\delta_{N}\right|=\mid L\left(P ; Q_{N-1}^{\prime}\right)-$ $L\left(P ; Q_{N}^{\prime}\right) \mid$; this approaches zero as $N \rightarrow \infty$. This may be seen by considering $L\left(P ; Q_{N-1}^{\prime}\right)$ $-L\left(P ; Q_{N}^{\prime}\right)$ as the discrete Green's function for the half-plane with boundary midway between $Q_{N-1}^{\prime}$ and $Q_{N}^{\prime}$.

From the uniform convergence of the series representing $G^{S}(P ; Q)$, we have that

$$
\Delta_{h} G^{S}(P ; Q)=\sum_{i=0}^{\infty}(-1)(-1)^{i} \delta\left(P ; Q_{i}\right) / h^{2}=-\delta(P ; Q) / h^{2} .
$$

Therefore, $G^{S}(P ; Q)$, as we have constructed it, is the discrete Green's function for the strip.

We now verify the estimate in (2). We have that

$$
\left|G^{S(m)}(P ; Q)\right| \leqq\left|\sum_{i=0}^{N}(-1)^{i} G^{H(m)}\left(P ; Q_{i}\right)\right|+\left|\sum_{i=N+1}^{\infty}(-1)^{i} G^{H(m)}\left(P ; Q_{i}\right)\right| .
$$

Let $W_{1}(N)$ (and $W_{2}(N)$ ) be the first (and second) summand in the expression above. Then we have that

$$
\left|W_{2}(N)\right| \leqq C \sum_{i=N+1}^{\infty} 1 / \rho_{P Q_{2 i}}^{m+1}=O(1 / N) .
$$

By simple rearrangements, we may write

where

$$
W_{2}^{(m)}(N)=V_{1}^{(m)}(N) \quad \text { and } \quad W_{2}^{(m)}(N)=V_{2}^{(m)}(N)
$$

$$
\begin{array}{rlrl}
V_{1}^{(m)}(N) & =\left|\sum_{i=0}^{(N-1) / 2}\left[G^{2 i}\left(P ; \xi, \eta_{2 i}\right)-G^{2 i}\left(P ; \xi,-\eta_{2 i}\right)\right]\right|, & N \text { odd, } \\
& =\left|\sum_{i=0}^{(N-2) / 2}\left[G^{2 i}\left(P ; \xi, \eta_{2 i}\right)-G^{2 i}\left(P ; \xi,-\eta_{2 i}\right)\right]+G^{H}\left(P ; \xi, \eta_{N}\right)\right|, & & N \text { even, }
\end{array}
$$


and

$$
\begin{array}{r}
V_{2}^{(m)}(N)=\left|G^{H}(P ; \xi, \eta)+\sum_{i=0}^{(N-2) / 2}\left[G^{2 i+1}\left(P ; \xi, \eta_{2 i+1}\right)-G^{2 j+1}\left(P ; \xi,-\eta_{2 j+1}\right)\right]\right|, \\
N \text { even, } \\
=\mid G^{H}(P ; \xi, \eta)+\sum_{j=0}^{(N-3) / 2}\left[G^{2 i+1}\left(P ; \xi, \eta_{2 j+1}\right)-G^{2 i+1}\left(P ; \xi,-\eta_{2 j+1}\right)\right] \\
-G^{H}\left(P ; \xi, \eta_{N}\right) \mid,
\end{array}
$$$$
N \text { odd, }
$$

where

$$
G^{i}\left(P ; \xi, \eta_{i}\right)=L\left(P ; \xi, \eta_{i+1}\right)-L\left(P ; \xi, \eta_{i}\right)
$$

and

$$
G^{i}\left(P ; \xi,-\eta_{j}\right)=L\left(P ; \xi,-\eta_{i+1}\right)-L\left(P ; \xi,-\eta_{i}\right) .
$$

We will now estimate the summands in $V_{1}^{(m)}(N)$ and $V_{2}^{(m)}(N)$. With the aid of Lemma 1.1, we have that

$$
\left|G^{2 i}\left(x, y ; \xi, \eta_{2 j}\right)-G^{2 i}\left(x, y ; \xi,-\eta_{2 i}\right)\right|=\left|h \sum_{z=-\nu}^{\nu-h} G_{\nu}^{2 j}\left(x, z ; Q_{2 i}\right)\right| .
$$

But

$$
\begin{aligned}
G^{2 i}\left(x, y ; \xi, n_{2 j}\right) & =G^{2 i}\left(x, y-\left(\eta_{2 i}+\eta_{2 i+1}\right) / 2 ; \xi, \eta_{2 i}-\left(\eta_{2 i}+\eta_{2 i+1}\right) / 2\right) \\
& =G^{H}\left(x, y^{\prime} ; \xi,\left(\eta_{2 j+1}-\eta_{2 j}\right) / 2\right)
\end{aligned}
$$

where $y^{\prime}=\left(\eta_{2 j+1}+\eta_{2 i}\right) / 2$. Hence,

$$
\left|G^{2 i(m)}\left(P ; Q_{2 j}\right)\right| \leqq B_{m}(a-\eta) / \rho_{P Q_{2 j}}^{m+1}, \quad\left|G^{2 i(m)}\left(P ; Q_{2 j}\right)\right| \leqq A_{m} / \rho_{P Q_{2 j}}^{m} .
$$

By a similar analysis, we have

$$
\left|G^{2 i+1(m)}\left(P ; Q_{2 j+1}\right)\right| \leqq B_{m} \eta / \rho_{P Q_{2 j+1}}^{m+1}, \quad\left|G^{2 j+1(m)}\left(P ; Q_{2 i+1}\right)\right| \leqq A_{m} / \rho_{P Q_{2 j+1}}^{m} .
$$

Therefore,

$$
\begin{array}{rlr}
\left|V_{1}^{(m)}(N)\right| & \leqq \sum_{i=1}^{(N-1) / 2} 2 y(a-\eta) B_{m+1} / \rho_{P Q_{2 i}}^{m+2}+2\left|G^{O(m)}\left(P ; Q_{0}\right)\right|, & N \text { odd, } \\
& \leqq \sum_{i=1}^{(N-2) / 2} 2 y(a-\eta) B_{m+1} / \rho_{P Q_{2} i}^{m+2}+2\left|G^{O(m)}\left(P ; Q_{0}\right)\right|+\left|G^{H(m)}(P ; Q)\right|, & N \text { even. }
\end{array}
$$

Observe that $G^{0}(P ; Q) \neq G^{H}(P ; Q)$ and $\left|G^{0}(x, y ; \xi, \eta)-G^{0}(x,-y ; \xi, \eta)\right| \leqq 2\left|G^{0}(P ; Q)\right|$. Now use the estimate

$$
\sum_{i=1}^{(N-1) / 2} 1 / \rho_{P Q_{2 i}}^{m+2} \leqq 1 / \rho_{P O}^{m+2}+\pi / 2 \sqrt{ } 2 a \rho_{P O}^{m+1},
$$

considering separately the cases $\rho_{P Q} \geqq a / 2$ and $\rho_{P Q}<a / 2$, to get 


$$
\left|V_{1}^{(m)}(N)\right| \leqq\left\{2 B_{m}+4.2 B_{m+1}\right\}(a-\eta) / \rho_{P Q}^{m+1}+O(1 / N) .
$$

By an analogous method of reasoning, we have

$$
\left|V_{2}^{(m)}(N)\right| \leqq\left\{B_{m}+9.5 B_{m+1}\right\} \eta / \rho_{P Q}^{m+1}+O(1 / N) .
$$

Combining our results, we have that

$$
\left|G^{S(m)}(P ; Q)\right| \leqq\left\{2 B_{m}+9.5 B_{m+1}\right\} \min \{\eta, a-\eta\} / \rho_{P Q}^{m+1} .
$$

This establishes (2).

We will now prove (3). If $\rho_{P Q} \geqq a$, then $\rho_{P Q} \geqq d(Q)$. Hence, $1 / \rho_{P Q}^{m} \geqq d(Q) / \rho_{P Q}^{m+1}$. If $\rho_{P Q}<a$, then

$$
\begin{aligned}
\left|\sum_{i=2}^{N} G^{H(m)}\left(P ; Q_{2 j}\right)-G^{H(m)}\left(P ; Q_{2 j+1}\right)\right| & \leqq\left|Q_{2 i+1}-Q_{2 i}\right| \sum_{j=1}^{\infty} A_{m} / \rho_{P Q_{2 j}}^{m+1} \\
& \leqq 2(a-\eta) A_{m} \pi / 2 \sqrt{ } 2 a \rho_{P Q}^{m} \leqq \pi A_{m} / \sqrt{ } 2 \rho_{P Q}^{m} .
\end{aligned}
$$

Therefore, (3) is proved with $J_{m}=\max \left\{\pi A_{m} / \sqrt{ } 2, H_{m}\right\}$.

We now proceed with (4). Here we need only show that $0 \leqq G^{S}(P ; Q) \leqq C d(Q) / \rho_{P Q}$ since $G^{S}(P ; Q)=G^{S}(Q ; P)$ by Lemma 1.1. Both of these estimates follow easily from the following result: If $\Delta_{h} H \leqq 0$ in $S_{h}$, if $\lim _{P \rightarrow \infty} H(P)=0$, and if $H(P) \geqq 0$ on $\partial S_{h}$, then $H(P) \geqq 0$ in $S_{h}+\partial S_{h}$. Now let $H=G^{H}(P ; Q)-G^{S}(P ; Q)$, with $G^{H}(P ; Q)$ the discrete Green's function for the upper half-plane $y=0$ or the lower half-plane $y=a$, to conclude the proof of the theorem.

3. Rectangular Regions. Let $R$ be the rectangular region determined by the vertices $(-c, 0),(b, 0),(b, a)$ and $(-c, a)$; here $a, b$ and $c$ positive numbers. We assume, with no loss in generality, that $a / h, b / h$ and $c / h$ are integers. Let $G(P ; Q)$ be the discrete Green's functions associated with $R_{h}$ and $\Delta_{h}$. We then have the following result.

THEOREM 3.1. If $i$ is a nonnegative integer, then we define $\xi_{i}$ to be the ith element of the sequence $0,2 b, 2 b+2 c, 4 b+2 c, 4 b+4 c, 6 b+4 c, 6 b+6 c, \cdots$, and if $i$ is $a$ negative integer, then we define $\xi_{i}$ to be the ith element of the sequence $-2 c,-(2 b+2 c)$, $-(2 b+4 c),-(4 b+4 c), \cdots$. Let $G^{S}(P ; Q)$ be the discrete Green's function for the strip $S_{h}$ determined by the lines $y=0$ and $y=a$. If we take $Q=(0, \eta)$ and $Q_{i}=$ $\left(\xi_{i}, \eta\right)$, then we have

$$
G^{R}(P ; Q)=\sum_{i=-\infty}^{\infty}(-1)^{i} G^{S}\left(P ; Q_{i}\right) .
$$

The case of the general $Q=(\xi, \eta)$ is handled by a simple translation. Moreover, there exist absolute constants $K_{m}, L_{m}$ and $C$ which depend upon the diameter of $R$, such that

$$
\begin{aligned}
& \left|D^{(m)} G^{R}(P ; Q)\right| \leqq K_{m} / \rho_{P Q}^{m}, \\
& \left|\tilde{D}^{(m)} G^{R}(P ; Q)\right| \leqq L_{m} d(Q) / \rho_{P Q}^{m+1},
\end{aligned}
$$

and

$$
G^{R}(P ; Q) \leqq C \min (d(P), d(Q)) / \rho_{P Q},
$$

where $d(X)$ is the distance of $X$ to $\partial R_{h}$.

Proof. We first show that the sequence in (1) is convergent. Let $i \geqq 0$. Then we 
easily have that $\lim _{i \rightarrow \infty} G^{S}\left(P ; Q_{i}\right)=0$. We also claim that $G^{S}\left(P ; Q_{i}\right) \geqq G^{S}\left(P ; Q_{i+1}\right)$ for every $P \in R_{h}$. To see this, let $T=\left\{(x, y):(x, y) \in S, x \leqq\left(\xi_{i}+\xi_{i+1}\right) / 2\right\}$. Along the line $x=\left(\xi_{i}+\xi_{i+1}\right) / 2$, we have that $G^{S}\left(P ; Q_{i}\right)=G^{S}\left(P ; Q_{i+1}\right)$. This is also true along the $\partial S_{h}$. Therefore, $G^{S}\left(P ; Q_{i}\right)-G^{S}\left(P ; Q_{i+1}\right) \geqq 0$ in $R_{h}$ by the extended monotonicity theorem which was stated at the end of the proof of Theorem 2.1. The series in (1) is therefore convergent as it is a monotonically decreasing alternating series with its terms tending to zero. A similar analysis applies to the case $i \leqq-1$.

Now we show that $G(P ; Q)=0$ for $P \in \partial R_{h}$. This is clear on $y=0$ or $y=a$. Let $P \in \partial R_{h}$ with $x=b$. Then

$$
G(P ; Q)=\sum_{i=0}^{n}(-1)^{i} G^{S}\left(P ; Q_{i}\right)+Q_{n+1}+\sum_{i=-1}^{-n^{\prime}}(-1)^{i} G^{S}\left(P ; Q_{i}\right)+R_{-n^{\prime}-1} .
$$

Now $\left|R_{n+1}\right| \leqq G^{S}\left(P ; Q_{n+2}\right)$ and $\left|R_{-n^{\prime}-1}\right| \leqq G^{S}\left(P ; Q_{-\left(n^{\prime}+1\right)}\right)$. Therefore,

$$
\begin{aligned}
G(P ; Q)= & G^{S}\left(P ; Q_{0}\right)-G^{S}\left(P ; Q_{1}\right)+\cdots+(-1)^{n+1}\left[G^{S}\left(P ; Q_{n+1}\right)-G\left(P ; Q_{-n}\right)\right] \\
& +\left(Q_{n+1}+Q_{-n-1}\right) .
\end{aligned}
$$

Now the midpoint of $\xi_{-n}$ and $\xi_{n+1}$ is $x=b$ and $G^{S}\left(P ; Q_{n+1}\right)=G^{S}\left(P ; Q_{-n}\right)$ along this line. Therefore, for $P=(b, y) \in \partial R_{h}$, we have $G(P ; Q)=0$. A similar analysis applies to the line $x=-c$.

By methods of Section 2, we easily have that $\Delta_{h} G(P ; Q)=-\delta(P ; Q) / h^{2}$.

We can also write $G^{R}(P ; Q)$ as the sum of the discrete Green's functions $G^{\prime}\left(P ; Q_{i}^{\prime}\right)$ for the strip $S_{h}^{\prime}$ bounded by the lines $x=-c$ and $x=b$. That is,

$$
G^{R}(P ; Q)=\sum_{i=-\infty}^{\infty}(-1)^{i} G^{\prime}\left(P ; Q_{i}^{\prime}\right)
$$

where $G^{\prime}\left(P ; Q_{i}^{\prime}\right)$ is the discrete Green's function for $S_{h}^{\prime}$ and $Q_{i}$ is an element of the sequence $\eta, 2 a-\eta, 2 a+\eta, 4 a-\eta, 4 a+\eta, \cdots$ for $i=0,1,2, \cdots$ and an element of the sequence $-\eta,-(2 a-\eta),-(2 a+\eta),-(4 a-\eta),-(4 a+\eta), \cdots$ for $i=$ $-1,-2,-3, \cdots$.

Now $\left|G^{\prime(m)}\left(P ; Q_{i}^{\prime}\right)\right| \leqq J_{m} d^{\prime}(Q) / \rho_{P Q_{i}}^{m+1}$, and $\left|G^{S(m)}\left(P ; Q_{i}\right)\right| \leqq J_{m} d(Q) / \rho_{P Q_{i}}^{m+1}$ where $d^{\prime}(Q)=\min (b, c), d(Q)=\min (\eta, a-\eta)$, and $\min \left(d^{\prime}(Q), d(Q)\right)$ is the distance from $Q$ to $\partial R_{h}$.

We also have the estimates, from the two different representations,

$$
\begin{aligned}
\left|G^{R(m)}(P ; Q)\right| & \leqq\left|G^{S(m)}(P ; Q)\right|+\left|\sum_{i=-\infty ; i \neq 0}^{\infty}(-1)^{i} G^{S(m)}\left(P ; Q_{i}\right)\right| \\
& \leqq\left|G^{\prime(m)}(P ; Q)\right|+\left|\sum_{i=-\infty ; i \neq 0}^{\infty}(-1)^{i} G^{\prime(m)}\left(P ; Q_{i}\right)\right|
\end{aligned}
$$

Now proceed as in the proof of Theorem 2.1 .

To prove (2.3), we may suppose that $d(P) \leqq d(Q)$. Then place a half-plane $H$ along the side of $\partial R_{h}$ whose distance to $P$ is minimal. The maximum principle shows that $G^{H}(P ; Q)$ dominates $G^{R}(P ; Q)$. Our proof is complete.

The results of this theorem are a significant improvement over the estimates in [5] where the order of the singularity in the $m$ th order difference quotient was $\rho_{P Q}^{-m-e}$ with $\epsilon>0$.

The rectangle formed by rotating the above rectangle through any integer multiple 
of $\pi / 4$ has a discrete Green's function which satisfies the same estimates as in Theorem 3.1.

In our next result, we obtain slightly stronger estimates on the discrete Green's function for a rectangle.

THEOREM 3.2. Estimates of the following type for the discrete Green's function over a rectangle, or triangle may be derived:

(a) $\left|G_{x}^{R}(P ; Q)\right| \leqq C d_{y}(P) d_{x}(Q) d_{y}(Q) / \rho_{P Q}^{4}$,

(b) $G^{R}(P ; Q) \leqq C d_{x}(P) d_{y}(P) d_{x}(Q) d_{y}(Q) / \rho_{P Q}^{4}$,

(c) $\left|G_{y}^{R}(P ; Q)\right| \leqq C d_{x}(P) d_{x}(Q) d_{y}(Q) / \rho_{P Q}^{4}$,

(d) $\left|G_{x \tilde{x}}^{R}(P ; Q)\right| \leqq C d_{y}(P) d_{x}(P) d_{x}(Q) d_{y}(Q) / \rho_{P Q}^{6}$,

(e) $\left|G_{y \bar{\nu}}^{R}(P ; Q)\right| \leqq C d_{x}(P) d_{x}(Q) d_{y}(Q) d_{x}(Q) / \rho_{P Q}^{6}$,

(f) $\left|G_{x y}^{R}(P ; Q)\right| \leqq C d_{x}(Q) d_{y}(Q) / \rho_{P Q}^{4}$,

where $d_{x}(P)$ or $d_{y}(P)$ is the distance, in the $x$ or $y$ direction, of $P$ to the boundary. Estimates of a similar type are valid for difference quotients in $Q$.

Proof. The argument proceeds briefly as follows:

(a) From Section 1 , we have $\left|G_{x \xi \eta}^{H}(P ; Q)\right| \leqq d_{\nu}(P) / \rho_{P Q}^{4}$. Hence

$$
\begin{aligned}
\left|G_{x \xi}^{S}(P ; Q)\right| & =\left|\sum_{j=0}^{\infty} G_{x \xi}^{H}\left(P ; Q_{i}\right)(-1)^{i}\right|=\left|\sum_{j=0}^{\infty}\left[h \sum_{Z_{j}=Q_{2 i}}^{Q_{2 j+1}} G_{x \xi \eta}^{H}\left(P ; Z_{i}\right)\right]\right| \\
& \leqq C \sum_{j=0}^{\infty} d_{\nu}(Q) d_{\nu}(P) / \rho_{P Q_{2 j}}^{4} \leqq C d_{\nu}(Q) d_{y}(P) / \rho_{P Q}^{4} .
\end{aligned}
$$

Now we obtain our result from the estimate

$$
\begin{aligned}
\left|G_{x}^{R}(P ; Q)\right| & =\left|\sum_{i=-\infty}^{+\infty} G_{x}^{S}\left(P ; Q_{i}\right)(-1)^{i}\right|=\left|\sum_{i=-\infty}^{+\infty}\left[h \sum_{W i=Q_{2 i}}^{Q_{2 i+1}} G_{x \xi}^{S}\left(P ; W_{i}\right)\right]\right| \\
& \leqq C \sum_{i=-\infty}^{+\infty} d_{x}(Q) d_{y}(Q) d_{\nu}(P) / \rho_{P Q_{2 i}}^{4} \leqq C d_{x}(Q) d_{\nu}(Q) d_{\nu}(P) / \rho_{P Q}^{4} .
\end{aligned}
$$

(b) Let $P^{\prime}$ be the point on $\partial R_{h}$ nearest $P$ in the $x$ direction. Then

$$
\begin{aligned}
G^{R}(P ; Q) & =G^{R}(P ; Q)-G^{R}\left(P^{\prime} ; Q\right) \leqq h \sum_{Z=P}^{P^{\prime}}\left|G_{x}^{R}(Z ; Q)\right| \\
& \leqq C \cdot d_{x}(P) d_{y}(P) d_{x}(Q) d_{y}(Q) / \rho_{P Q}^{4} .
\end{aligned}
$$

The remaining parts of the theorem are proved similarly.

The above results may be used to improve Theorem 1.1 as seen in the next theorem.

THEOREM 3.3. Estimates of the following type, for the discrete Green's function over a half-plane, are valid:

(a) $G^{H}(P ; Q) \leqq C d(Q) d(P) / \rho_{P Q}^{2}$,

(b) $\left|G_{x}^{H}(P ; Q)\right| \leqq C d(Q) d(P) / \rho_{P Q}^{3},\left|G_{\xi}^{H}(P ; Q)\right| \leqq C d(Q) d(P) / \rho_{P Q}^{3}$, etc.,

(c) $\left|G_{x \bar{x}}^{H}(P ; Q)\right| \leqq C d(P) d(Q) / \rho_{P Q}^{4},\left|G_{x \xi}^{H}(P ; Q)\right| \leqq C d(P) d(Q) / \rho_{P Q}^{4}$, etc.

Here $C$ is an absolute constant which is independent of $h$.

Proof. As an example of the method of proof we will establish (c). Let $R$ be a square in the half-plane $H_{h}$ one side of which is coincident with the boundary of $H_{h}$. Construct $R_{h}$ such that the distance from $P$ or $Q$ to the three sides of $R_{h}$, none of which is on $\partial H_{h}$, is greater than $\max \left(d(P), d(Q), \rho_{P Q}\right)$; here $d(X)$ is the distance from $X$ to the $\partial H_{h}$. Then 


$$
G_{x \dot{x}}^{R}(P ; Q)-G_{x \dot{x}}^{H}(P ; Q)=h \sum_{Z \in \partial R_{h^{\prime}}} G^{H}(Z ; Q) G_{n x \dot{x}}^{R}(P ; Z)
$$

where $\partial R_{h}^{\prime}=\partial R_{h}-\left(\partial R_{h} \cap \partial H_{h}\right)$ and the subscript $n$ denotes a normal difference quotient with respect to the moving variable. Hence,

$$
\left|G_{x \dot{x}}^{R}(P ; Q)-G_{x \bar{x}}^{H}(P ; Q)\right| \leqq C d(Q) d(P) / \rho_{P Q}^{4},
$$

and

$$
\left|G_{x \dot{x}}^{H}(P ; Q)\right| \leqq C d(Q) d(P) / \rho_{P Q}^{4} .
$$

Many additional properties of the discrete Green's function may be simplified by our next result.

THEOREM 3.4. Over a rectangle $R_{h}$, the discrete Green's function $G^{R}(P ; Q)$ satisfies:

$$
G_{x \dot{x}}^{R}(P ; Q)=G_{\xi \bar{\xi}}^{R}(P ; Q) \quad \text { and } \quad G_{\nu \bar{\nu}}^{R}(P ; Q)=G_{\eta \dot{\eta}}^{R}(P ; Q) \text {. }
$$

Proof. Let $U(Z)=G_{x \dot{x}}^{R}(Z ; Q)$ and $V(Z)=G^{R}(P ; Z)$. Since we may make, by reflection, $U(Z)=0$ on $\partial R_{h}$, our result follows by an application of the discrete Green's identity; see [5].

The above results are apparently not valid over other simple regions such as bounded $L$-shaped regions or knife-shaped regions.

We will now state a final improvement of earlier results; the proof is similar to that of Theorem 3.3.

THEOREM 3.5. Estimates of the following type are valid for the discrete Green's function over an infinite strip.

(a) $0 \leqq G^{S}(P ; Q) \leqq C d(Q) d(P) / \rho_{P Q}^{2}$,

(b) $\left|G_{x}^{S}(P ; Q)\right| \leqq C d(Q) d(P) / \rho_{P Q}^{3},\left|G_{y}^{S}(P ; Q)\right| \leqq C d(Q) d(P) / \rho_{P Q}^{3},\left|G_{\xi}^{S}(P ; Q)\right| \leqq$ $C d(Q) d(P) / \rho_{P Q}^{3},\left|G_{\eta}^{S}(P ; Q)\right| \leqq C d(Q) d(P) / \rho_{P Q}^{3}$,

(c) $\left|G_{x \bar{x}}^{S}(P ; Q)\right| \leqq C d(P) d(Q) / \rho_{P Q}^{4},\left|G_{x y}^{S}(P ; Q)\right| \leqq C d(P) d(Q) / \rho_{P Q}^{4},\left|G_{x \xi}^{S}(P ; Q)\right| \leqq$ $C d(P) d(Q) / \rho_{P Q}^{4},\left|G_{\nu \eta}^{S}(P ; Q)\right| \leqq C d(P) d(Q) / \rho_{P Q}^{4}$, etc.,

where $d(P)$ is the distance of $P$ to the boundary of $S_{h}$ and $C$ is a generic constant independent of $h$.

4. General Domains. Let $\Omega$ be a plane region. Place a square grid on the plane with grid width $h$. We say that a grid point $P \in \Omega_{h}$ if $P$ and the four grid neighbors of $P$ are in $\Omega$. Let $\partial \Omega_{h}$ be those grid points which are in $\bar{\Omega}$ but not in $\Omega_{h}$.

Let $h_{n}$ be some sequence tending monotonically to zero as $n$ tends to $\infty$. Then we call $\Omega$ a discrete $h$-convex set if for each $n$ and for each $P \in \partial \Omega_{h_{n}}$ at least one of the lines through $P$, which is parallel to a coordinate axis or makes an angle of $\pi / 4$ with a coordinate axis, has the entire set $\Omega_{h_{n}}$ to one side of this line. Examples of discrete $h$-convex sets are triangles, rectangles, circles, ellipses and knife-shaped regions (e.g. the region formed by the coordinates $(0,0),(c, 0),(c, 2 c)$ and $(0, c))$.

The concept of discrete $h$-convex is essential for our estimates in this section. We shall assume that our regions satisfy this condition and, when we write $\Omega_{h}$, we mean an element of the sequence $\left\{\Omega_{h_{n}}\right\}$ where the sequence $\left\{h_{n}\right\}$ is that sequence used in the definition.

We remark that the estimates we have obtained to date hold for half-planes, quarter-planes, eighth-planes, strips, triangles and rectangles.

We will now state and outline the proof of our first result. 
THEOREM 4.1. Let $\Omega$ be a discrete $h$-convex set and let $G(P ; Q)$ be the discrete Green's function associated with $\Omega_{h}$ and $\Delta_{h}$. Then there exist absolute constants $M_{0}$, $M_{1}, N_{0}, N_{1}$, all independent of $h$, such that

(1)

$$
\begin{aligned}
|G(P ; Q)| \leqq M_{0} & \left\{\begin{array}{c}
d(P) \\
o r \\
d(Q)
\end{array}\right\} / \rho_{P Q} ; \quad|G(P ; Q)| \leqq N_{0} d(P) d(Q) / \rho_{P Q}^{2} \text { and } \\
\left|G^{(1)}(P ; Q)\right| \leqq M_{1} / \rho_{P Q} ; & \left|G^{(1)}(P ; Q)\right| \leqq N_{1} d(P) / \rho_{P Q}^{2} .
\end{aligned}
$$

Proof. We take the discrete Green's function for a half-plane determined by any point $P \in \partial \Omega_{h}$. The Monotonicity Theorem and the results of Section 3 give the estimate in (3.1).

For the estimate in (3.2), we proceed as follows:

Case 1. $\min \{d(P), d(Q)\} \geqq \rho_{P Q}$.

Construct, about $Q$, a square $S_{h}$ of (approximate) sidelength $\rho_{P Q}$. If this is not possible, as in the case $\rho_{P Q}=h$, construct the square of sidelength $2 h$ or $3 h$; our argument will proceed in a similar way. Let $\bar{G}(P ; Q)$ be the discrete Green's function for $S_{h}$. We will use the following form of the discrete Green's formula:

$$
h^{2} \sum_{R \in S_{h}}\left\{U(R) \Delta_{h} R(R)-V(R) \Delta_{h} U(R)\right\}=h \sum_{R \in \partial S_{h}}\left\{U(R) V_{n}(R)-V_{n}(R) U(R)\right\} .
$$

Substitute $\bar{G}(R ; Q)=U(R)$ and $G(P ; R)=V(R)$ to get the representation

$$
G(P ; Q)=-h \sum_{R \in \partial S_{h}} \bar{G}_{n}(R ; Q) G(P ; R) .
$$

Hence,

$$
\begin{aligned}
\left|G^{(1)}(P ; Q)\right| & =\left|h \sum_{R \in \partial S_{h}} \bar{G}_{n}^{(1)}(R ; Q) G^{Q}(P ; R)\right| \\
& \leqq \max _{R}\left\{C \cdot h\left(\rho_{P Q} / h\right)\left(d(R) / h \rho_{P Q}^{2}\right)\left(d(P) / \rho_{P Q}\right)\right\} \leqq N_{1} d(P) / \rho_{P Q}^{2}
\end{aligned}
$$

Also, we have that

$$
\begin{aligned}
|G(P ; Q)| & \leqq \max _{R}\left\{C h\left(\rho_{P Q} / h\right)\left(d(R) d(Q) / h \rho_{P Q}^{2}\right)\left(d(P) / \rho_{P Q}\right)\right\} \\
& \leqq M_{1} d(P) d(Q) / \rho_{P Q}^{2} .
\end{aligned}
$$

Case 2. $\min \{d(P), d(Q)\}<\rho_{P Q}$.

If $d(Q) \geqq \rho_{P Q}$, then we will proceed as in the case above.

If $d(Q)<\rho_{P Q}$, construct a square $S_{h}^{\prime}$ of sidelength $d(Q)$ about $Q$. Now let $S_{h}$ be the region formed by the intersection of $\Omega_{h}$ and $S_{h}^{\prime}$. If we have $d(Q) \leqq \rho_{P Q} / 2$, the boundary of $S_{h}^{\prime}$ and $\partial \Omega_{h}$ are coincident. From (2), we obtain the estimate

$$
\begin{aligned}
\left|G^{(1)}(P ; Q)\right| & =\left|-h \sum_{R \in \partial S_{h}} \bar{G}^{(1)}(R ; Q) G(R ; P)\right| \\
& <\max _{R}\left\{h C(d(Q) / h)\left(d\left(R^{\prime}\right) / h \rho_{R Q}^{2}\right)\left(d(R) d(P) / \rho_{R P}^{2}\right)\right\} \leqq N_{1} d(P) / \rho_{P Q}^{2},
\end{aligned}
$$

where we observe that $d(R) \leqq 2 d(Q)$ and $\left|\bar{G}_{n}^{(1)}(R ; Q)\right|=\left|\bar{G}^{(1)}\left(R^{\prime} ; Q\right) / h\right|$, with $R^{\prime}$ a grid point $h$ units from the boundary of $S_{h}$. Our proof is easily completed. 
At this point, we remark that the estimates in (1.2) were obtained as a result of the estimates in (1.1). The estimates in (1.1) were completely dependent on the assumption that $\Omega$ is a discrete $h$-convex set.

In our next result, we will see that an obtuse corner on the $\partial \Omega_{h}$ produces a complication in establishing estimates on difference quotients for general regions. These complications are present in the continuous theory but not quite as bad as our estimates predict in the discrete case; see [4]. This situation seems to indicate that our estimates may only be slightly improved; at least with reference to the five-point approximation of $\Delta$.

THEOREM 4.3. Let $\Omega_{h}$ be a discrete h-convex set and let $G(P ; Q)$ be the discrete Green's function for $\Delta_{h}$ over $\Omega_{h}$. Then there exists an absolute constant $N$ such that

$$
\left|G^{(2)}(P ; Q)\right| \leqq N d(Q) / d^{\prime}(P) \rho_{P Q}^{2},
$$

where $d^{\prime}(P)$ is the minimum of $\rho_{P Q}$ and the distance of $P$ to the nearest obtuse angle of $\Omega_{h}$.

Proof. The argument proceeds as in the proof of Theorem 4.1. Construct a square $R_{h}$, with center $P$, of (approximate) sidelength $\rho_{P Q}$. Extend $G(P ; Q)$ to all of $R_{h}$ by reflection. This is always possible if $R_{h}$ does not contain the vertex of an abtuse angle. By the use of Green's Theorem, we have

$$
G(P ; Q)=h \sum_{Z} G_{n}^{R}(P ; Z) G(Z ; Q) .
$$

Thus,

$$
\begin{aligned}
\left|G^{(2)}(P ; Q)\right| & \leqq h \sum_{Z \in \partial R_{h}}\left|G_{n}^{R(2)}(P ; Z)\right| G(Z ; Q) \\
& \leqq N \cdot h \sum_{Z \in \partial R_{h}}\left(1 / \rho_{P Z}^{3}\right)\left(d(Q) / \rho_{Z Q}\right) \leqq N d(Q) / \rho_{P Q}^{3} .
\end{aligned}
$$

If $R_{h}$ contains a vertex of an obtuse angle, a discrete harmonic extension of $G(P ; Q)$ to all of $R_{h}$ is impossible. Hence, we construct a square of sidelength equal to $d^{\prime}(P)$ the distance of $P$ to the vertex of the obtuse angle. We then have

$$
\left|G^{(2)}(P ; Q)\right| \leqq N h \sum_{Z \in \partial R_{\Lambda}}\left(1 / \rho_{P Z}^{3}\right)\left(d(Q) d(Z) / \rho_{Z Q}^{2}\right) \leqq N d(Q) / d^{\prime}(P) \rho_{P Q}^{2} .
$$

This follows from the fact that the perimeter of the square is $4 d^{\prime}(P)$ and $d(Z)<3 d^{\prime}(P)$.

Combining these two results, the theorem follows.

5. Discrete Green's Functions and Variable Coefficients. Let $R$ be a rectangular region with one side parallel to a coordinate axis. Let $G(P ; Q)$ be the solution to the problem

$$
\begin{aligned}
a(P) G_{x \bar{x}}(P ; Q)+c(P) G_{y \bar{\nu}}(P ; Q) & =-\delta(P ; Q) / h^{2}, & & P \in R_{h}, \\
G(P ; Q) & =0, & & P \in \partial R_{h},
\end{aligned}
$$

where difference quotients in (1) are with respect to the components of $P$ and where we assume that, for all $P \in \bar{R}$, there exist positive constants $\lambda$ and $L$ such that

$$
\lambda \leqq\{a(P), c(P)\} \leqq L .
$$

In this section, we shall indicate how we may extend the analysis of the preceding sections to obtain estimates on the solution to (1). We shall only consider rectangular 
domains; modifications necessary to extend the results of Section 4 will be clear from this case.

Our estimates, in this section, on the orders of growth of difference quotients of the solution to (1) will be an improvement and an extension of the results in [5, p. 31]. Our proof will rest heavily on the method of proof in [5, Theorem 3]. We will also use a result of Bramble and Thomée [1, Theorem, p. 585] on the rate of growth of $G(P ; Q)$; in particular, their result says that $\{G(P ; Q)\}^{p}$ is summable for any power $p \geqq 0$.

THEOREM 5.1. Let $G(P ; Q)$ be the solution to $(1)$. If $a(P)$ and $c(P)$ are $\alpha$-Hölder continuous over $\bar{R}$ with common Hölder constant $L_{\alpha}$ and if the condition in (2) is satisfied, then there exist constants $S_{m}$ and $T_{m}$, which depend upon $L, \lambda, L_{\alpha}$, diam $R$ and $\alpha$ but are independent of $h$, such that

$$
\left|D^{(m)}(P ; Q)\right| \leqq S_{m} / \rho_{P Q}^{m} ; \quad\left|\tilde{D}^{(m)}(P ; Q)\right| \leqq T_{m} \min \{d(P), d(Q)\} / \rho_{P Q}^{m+1} .
$$

Proof. We reflect $G(P ; Q)$ into a region $\tilde{\Omega}_{h}^{\prime} \supset \bar{\Omega}$ with $\tilde{\Omega}_{h}^{\prime}$ described in [5]. About $Q \in \Omega_{h}$ and each of its reflected images, we construct squares $M_{h}(Q)$ of sidelength $N_{0} h$ where $N_{Q}$ is independent of $h$ and $Q$. Let $P_{0} \in \Omega_{h}$ but not in any of these squares $M_{h}(Q)$. About $P_{0}$ construct a square $K_{h}\left(P_{0}\right) \subset \tilde{\Omega}_{h}^{\prime}-\{Q\}$ where $\{Q\}$ is the set $Q$ and its reflected images. Let $C_{1}$ and $C_{2}$ be positive numbers in $(0,1)$ such that

$$
\rho_{P_{0} Q} \geqq C_{2} \rho_{P_{0} Q} \geqq \operatorname{diam}\left(K_{h}\left(P_{0}\right)\right) \geqq C_{1} \rho_{P_{0} \odot}
$$

and, for every $R \in K_{h}\left(P_{0}\right)$,

$$
\rho_{R Q} \geqq\left(1-C_{2}\right) \rho_{P_{0} Q} ;
$$

note that $N_{Q}$ will depend on $C_{1}$ and $C_{2}$. Let $G^{\prime}(P ; Q)$ be the solution to the problem

$$
\begin{aligned}
a\left(P_{0}\right) G_{x \grave{x}}^{\prime}(P ; Q)+c\left(P_{0}\right) G^{\prime}(P ; Q) & =-\delta(P ; Q) / h^{2}, & & P \in K_{h}\left(P_{0}\right), \\
G^{\prime}(P ; Q) & =0, & & P \in \partial K_{h}\left(P_{0}\right) .
\end{aligned}
$$

Then we have the representation

$$
G(P ; Q)=h^{2} \sum_{W \in K_{h}\left(P_{0}\right)} G^{\prime}(P ; W) F(W)+H(P),
$$

where $F(W)=\left[a\left(P_{0}\right)-a(W)\right] G_{x \dot{x}}(W ; Q)+\left[c\left(P_{0}\right)-c(W)\right] G_{y \dot{\nu}}(W ; Q)$ and $H(P)$ solves the problem $a\left(P_{0}\right) H_{x \bar{x}}(P)+c\left(P_{0}\right) H_{y \bar{y}}(P)=0$ for $P \in K_{h}\left(P_{0}\right)$ and $H(P)=G(P ; Q)$ for $P \in \partial K_{h}\left(P_{0}\right)$. Now we may estimate difference quotients of the solution to (6), as we did in Theorem 3.1, but now we must account for the coefficients; note that if $L(x-\xi, y-\eta)$ is as defined in (1) of Section 1 but with a ch $\mu+c \cos \lambda=a+c$, then the discrete Green's function for the operator in (6) over $\pi^{+}$is given by $\{L(x-\xi, y+\eta)-L(x-\xi, y-\eta)\} / a$.

Let

$M_{2}(G: P ; Q)$

$$
\equiv \max \left\{\rho_{P Q}^{2}\left|G_{x \bar{x}}(P ; Q)\right|, \rho_{P Q}^{2}\left|G_{y \dot{y}}(P ; Q)\right|, \rho_{P Q}^{2}\left|G_{x y}(P ; Q)\right|: P, Q \in R_{h}\right\} .
$$

Suppose the diam $R$ is so small that

$$
\text { 12(diam } R)^{\alpha} H_{\alpha}\left(1+N_{0}^{2}\right) K_{2} \leqq \alpha
$$

where $K_{2}$ is derived from (6) as in Theorem 3.1. Then we may estimate $M_{2}(G: P ; Q)$ and prove our theorem. 
Now let us remove the constraint in (8). Let $R$ be a rectangular domain. Then $M_{2}(G: P ; Q)$ occurs at some point in $\bar{R}_{h}$; call the point $P_{0}$. About $P_{0}$ draw a square of diameter equal to $\min \left(d_{0}, \rho_{P_{0} Q} / 2\right)$ where $d_{0}$ is a number which when substituted for diam $R$ in (8) produces an equality. Our theorem now follows.

Center for the Application of Mathematics

Lehigh University

Bethlehem, Pennsylvania 18015

1. J. H. Bramble \& V. Thomée, "Pointwise bounds for discrete Green's functions," SIAM J. Numer. Anal., v. 6, 1969, pp. 583-590. MR 41 \#7870.

2. D. F. DeSanto \& H. B. Keller, "Numerical studies of transition from laminar to turbulent flow over a flat plate," J. Soc. Indust. Appl. Math., v. 10, 1962, pp. 569-595. MR $28 \# 800$.

3. C. R. Deeter \& G. Springer, "Discrete harmonic kernels," J. Math. Mech., v. 14, 1965 , pp. 413-438. MR 34 \#970.

4. R. Sherman Lehman, "Developments at an analytic corner of solutions of elliptic partial differential equations," J. Math. Mech., v. 8, 1959, pp. 727-760. MR 21 \#4291.

5. G. T. MCAllister, "A priori bounds on difference quotients of solutions to some linear uniformly elliptic difference equations," Numer. Math., v. 11, 1968, pp. 13-37. MR 37 $\# 2465$.

6. G. T. MCAllister, "An application of a priori bounds on difference quotients to a constructive solution of mildly quasilinear Dirichlet problems," J. Math. Anal. Appl., v. 24, 1968, pp. 582-607. MR 38 \#2963.

7. W. H. McCrea \& F. J. W. Whipple, "Random paths in two and three dimensions," Proc. Roy. Soc. Edinburgh, v. 60, 1940, pp. 281-298. MR 2, 107.

8. Moshe MANGAD, "Bounds for the two-dimensional discrete harmonic Green's function," Math. Comp., v. 20, 1966, pp. 60-67. MR 33 \#6856.

9. J. Nitsche \& J. C. C. Nitsche, "Error estimates for the numerical solution of elliptic differential equations," Arch. Rational Mech. Anal., v. 5, 1960, pp. 293-306. MR 22 \#8664.

10. R. B. Simpson, "A fundamental solution for a biharmonic finite-difference operator," Math. Comp., v. 21, 1967, pp. 321-339. MR 37 \#2466.

11. F. STUMMEL, "Elliptische Differenzenoperatoren unter Dirichlet Randbedingungen," Math. Z., v. 97, 1967, pp. 169-211. MR 36 \#346.

12. KJELL-Ove WIDMAN, "Inequalities for the Green function and boundary continuity of the gradient of solutions of elliptic differential equations," Math. Scand., v. 21, 1967, pp. 17-37. MR 39 \#621. 